\title{
Noisy three-player dilemma game: robustness of the quantum advantage
}

\author{
Pranav Kairon $^{1} \cdot$ Kishore Thapliyal $^{2} \cdot$ R. Srikanth ${ }^{3} \cdot$ Anirban Pathak $^{4}$ \\ Received: 12 April 2020 / Accepted: 18 August 2020 / Published online: 31 August 2020 \\ (c) Springer Science+Business Media, LLC, part of Springer Nature 2020
}

\begin{abstract}
Games involving quantum strategies often yield higher payoff. Here, we study a practical realization of the three-player dilemma game using the superconductivity-based quantum processors provided by IBM Q Experience. We analyze the persistence of the quantum advantage under corruption of the input states and how this depends on parameters of the payoff table. Specifically, experimental fidelity and error are observed not to be properly anti-correlated; i.e., there are instances where a class of experiments with higher fidelity yields a greater error in the payoff. Further, we find that the classical strategy will always outperform the quantum strategy if corruption is higher than $50 \%$.
\end{abstract}

Keywords Quantum game - Effect of noise on quantum advantages · Three-party dilemma game $\cdot$ Experimental realization of a quantum game

\section{Introduction}

Game theory provides a way to learn about decisive communication between rational and self-seeking agents. Therefore, it plays an important role in the fields of computer science, economics, biology, psychology, etc. (see [1,2] for review articles). Computationally, game theory can be used to model algorithms $[3,4]$ as well as to check

Anirban Pathak

anirban.pathak@jiit.ac.in

Kishore Thapliyal

kishore.thapliyal@upol.cz

1 Delhi Technological University, Bawana Road, Delhi 110042, India

2 RCPTM, Joint Laboratory of Optics of Palacky University and Institute of Physics of Academy of Science of the Czech Republic, Faculty of Science, Palacky University, 17. listopadu 12, 771 46 Olomouc, Czech Republic

3 Poornaprajna Institute of Scientific Research, Bengaluru, Karnataka 560080, India

4 Jaypee Institute of Information Technology, A-10, Sector-62, Noida UP-201309, India 
the robustness of networks and corresponding attack strategies [5]. In cryptography, the communication task can be visualized as a game between the parties trying to communicate securely and an eavesdropper (see [6] and references therein). With the advent of quantum computing, it is observed that resources used in quantum computing, such as quantum coherence and entanglement, provide alternative solutions to classical games.

We may mention, for example, the emergence of cooperation in the prisoner's dilemma game [7] and the resolution of the coordination in battle of sexes game [8] using entanglement. Specifically, as all the players wish to maximize their gain or payoff in games, for which the umpire has laid down the rule(s), players using quantum mechanical tactics are found to attain a higher payoff compared to the classical one [9]. Further, the dilemma disappears in prisoner's dilemma with the use of quantum resources under unitary operations $[10,11]$. Along the same line, optimal cloning of quantum states is also studied as game [12]. Quantum games based on monogamy of entanglement are shown to be useful in device-independent quantum cryptography [13]. Our understanding of several other foundational aspects of quantum mechanics is improved by considering games, such as nonlocality [14], the uncertainty bound on nonlocality [15], contextuality [16], PR-boxes [17], as well as applications in quantum reinforcement learning [18] and quantum machine learning [19].

Over the course of time, multiplayer quantum games were also introduced that exploit quantum correlation to prevent betrayal by individual players [20]. It has been suggested that these quantum games may shed light on the interactions in manyparticle systems [21]. One such multiplayer game is the three-party counterpart of the prisoner's dilemma. In the classical version, all three players prefer to choose strategies analogous to the corresponding two-party case. The dilemma exists because the Nash equilibrium does not coincide with the Pareto optimal [20]. Specifically, a Nash equilibrium is the situation in which no participant can gain by a unilateral change of strategy, while Pareto optimal corresponds to the situation that any change in strategy would make at least one individual worse off [20]. Still in quantum case, the use of tripartite entanglement shows certain advantage. Moreover, computing the Nash equilibrium in the three- and four-player games is shown to be a hard problem $[22,23]$. An experimental verification of three-player dilemma game using NMR was reported in [24]. In the recent past, other games have been realized on photonic quantum computer [25-28] and ion trap platform [29].

In general, the dilemma games are relevant in several studies of biology, economics, psychology, international relations and sports, to name a few. For instance, King Solomon's dilemma [30] based on the Old Testament can model prize allocation, research grant distribution, etc. Another multiparty version of prisoner's dilemma is diner's dilemma in which each player has to choose whether to order an expensive or an inexpensive dish if they have to equally share the bill [31]. An iterated version of this diner's dilemma game is useful in the social dynamics of networks and situational awareness. Such iterated multiparty prisoner's dilemma in the context of social dynamics is discussed in the past, too [32]. Along the same line, dilemma of the players in other games is used to introduce the conditional probability [33].

Decoherence is the Achilles' heel of quantum computing and information processing in particular, and technology in general. Similar results are shown for the quantum 
games [34]. Independently, the effect of errors in the initial state preparation (as corruption by a demon) on the outcome of three-player dilemma game is studied assuming that the players are unaware of corruption and that there is no decoherence [21]. Interestingly, beyond a pivotal value of corruption, it can be observed that players fare off better with the classical strategies, but since players have no knowledge of the level of corruption, they have to stick to their original strategies. Furthermore, a quantum game reduces to classical game if one of the parties allows his qubit to decohere under Markovian noise channels [35], while Nash equilibria are unchanged by decoherence for prisoner's dilemma [36].

Here, we wish to implement the three-player dilemma game [20] on IBM quantum computer ${ }^{1}$ and study how the change in the utility function affects the point of quantum advantage. Interestingly, this is the first realization of a game with corrupt source on a superconducting qubits-based quantum computer. Despite high error rate and the limited qubit connectivity, IBM quantum computers have been shown to run a wide array of algorithms (see [37,38] and references therein). Thus, we realize the game on IBM Q Experience and compare the experimental payoffs with previous experiments on NMR [24]. On generalizing the payoff table in the noisy game, the point where quantum advantage disappears also changes which leads to some interesting observations. In specific, they show how robust the quantum strategy is. An application of these results is that given a known corruption level, the payoff table (the relative stakes) may, in a range, be chosen to give an advantage to the quantum strategy. Finally, we show that classical strategies dominate when corruption is higher than $50 \%$ in the proposed game.

The rest of the paper is organized as follows. We introduce three-player quantum dilemma in Sect. 2. The noisy counterpart of the game and its experimental implementation are discussed in Sect. 3. We further discuss all the results in detail in the penultimate section before concluding the paper in Sect. 5 .

\section{Three-player quantum dilemma}

Before we formally describe three-player quantum dilemma, it will be apt to state the notion of game in general and quantum game in particular. A game can be defined as the set $S=$ \{players $P_{j}$, their actions $\sigma_{j}^{k}$, outcomes $O_{l}$, payoffs $p_{k}$ \}. To solve a game consists in determining the optimal strategies for all players, where a strategy refers to a player's move given the information available to her. A quantum game is a game where the player's actions correspond to applying a quantum operation on a joint state shared by all players, and in principle, even superposition of players' actions is allowed.

The multiparty dilemma game, generalizing the two-player prisoners' dilemma, was introduced as a multiparty counterpart of the prisoner's dilemma game, where each person has two choices: either to cooperate (0) or defect (1). The three-player dilemma resembles El Farol Bar problem that players have to decide independently

\footnotetext{
1 https://www.research.ibm.com/ibm-q/. Accessed on September 2019
} 


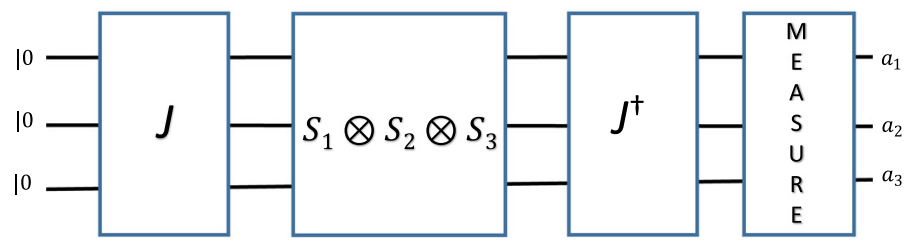

Fig. 1 (Color online) Circuit diagram that can be used for the realization of the three-player quantum dilemma game. Here, the measurement outcomes in the $Z$ basis are shown as $a_{j} \in\{0,1\}$

whether to go or not to a bar with seating capacity for only two (see [24] and references therein).

In the three-player quantum dilemma game, each player is provided one qubit by the umpire, who performs an entangling operation on state $|000\rangle$ before that which increases the nonclassical correlation among the players. The entangling gate $J$ can be defined as in Ref. [20]:

$$
J=\cos \frac{\gamma}{2} \mathrm{ID}^{\otimes 3}+i \sin \frac{\gamma}{2} X^{\otimes 3}
$$

where ID and $X$ are identity and Pauli NOT gates, respectively. Without loss of generality, we choose the case when the correlation is maximum, i.e., $\gamma=\pi / 2$. Further, it can be checked that for minimum correlation, i.e., $\gamma=0$, the game reduces to its classical counterpart [11].

In quantum game, each person is allowed to choose an operation from a strategy set $S$, consisting of three elements $S=\left(S_{1}, S_{2}, S_{3}\right)$, where $S_{1}=X$ means the player wants to attend the party; $S_{2}=H$ corresponds to the player's choice to go with half a probability; and $S_{3}=$ ID represents the player wants to stay at home. Note that the choice of $S_{2}$ does not have a counterpart in classical games. As far as a classical player in game theory is considered, he is allowed to choose his operations probabilistically in mixed strategy. In the present context, it is already shown that a classical player remains with unchanged dominant strategy even when allowed to select mixed strategies (for more details, see [21]). However, in what follows, we will observe that $S_{2}$ plays a significant role in the present quantum game. This is a restricted strategy set (as there can be an infinitely many possible quantum strategies each corresponding to a different unitary operation), but it encompasses all the nonclassical characteristics we want to demonstrate through this game. Subsequently, a disentangling operation $J^{\dagger}=\frac{\mathrm{ID}^{\otimes 3}-i X^{\otimes 3}}{\sqrt{2}}$ is performed before measuring in the computational basis. The circuit diagram of the game is shown in Fig. 1.

Thus, when none of the players decides to go, i.e., the measurement outcome is $|000\rangle$ (represented by corresponding bit values 000 in Table 1), nobody is happy since they could not attend the party but are not sad since none of the friends betrayed, and thus everybody gets 0 payoff. However, if one person decides to go, then the other two will be unhappy (with payoff $-n$ ), and the one attending the party does not enjoy being alone (with payoff $p$ ). When two of the friends decide to go, they both fare off with $n$ payoff each since they get to go to the party with company, while the friend 
Table 1 Generalized payoff table for three-player dilemma game depending upon the possible measurement outcomes

\begin{tabular}{ll}
\hline Bit values corresponding to possible measurement outcome $a_{1} a_{2} a_{3}$ & Payoffs $(\$)$ \\
\hline 000 & $0,0,0$ \\
001 & $-n,-n, p$ \\
010 & $-n, p,-n$ \\
011 & $p, n, n$ \\
100 & $p,-n,-n$ \\
101 & $n, p, n$ \\
110 & $n, n, p$ \\
111 & $q, q, q$ \\
\hline
\end{tabular}

In previous adaptations of the game [20], $n=9, p=1$ and $q=2$ were used

left behind is not too dejected since his presence would have overcrowded the party so he gets $p$. If all of them decide to go, they get payoff of $q$ each since their presence has overcrowded the party. Accordingly, we impose $n>q>p>0$.

This problem is also relevant in the virus and social distancing requirement during pandemics, such as the current coronavirus situation. For example, to enthuse people to brave the situation and pay a visit, a restaurant may make an attractive offer. However, to reduce the probability of the transmission of the viral infection, if too many people arrive, it will have to turn away some in order to allow only two people to sit per table as per social distancing norms.

Yet another example for the three-player dilemma that members of the academic would more readily relate to would be the dilemma of three academic collaborators in applying for a research grant. If two of them apply, they are likely to receive the grant, whereas they probably would receive insufficient or no funding if all three apply for it. Also, they would not be happy if none of them applies or their collaborator gets it but not them. The dilemma shown previously was by considering $n=9, p=1$, and $q=2$ [20]. Here, we study a general description of such payoff tables and show how the payoff depends on these parameters in the noisy game (subjected to constraint $0<p<q<n)$. One motivation for this is to understand whether and how the game's stakes can be fixed based on knowledge of the preparation noise in the system. In [20], it is shown that in a special case considering a different set of values of the payoffs, quantum players do not have any advantage over the classical strategy which is no longer a Nash equilibrium. Therefore, we have restricted ourselves to the aforementioned constraint which ensures that classical Nash equilibrium exists. To the best of our knowledge, this is the first attempt to generalize the payoff table for the three-player dilemma game in analogy of prisoner's dilemma [39].

\section{IBM implementation and noisy state preparation}

For the given strategy space $S$, there are three choices per player which gives us $3^{3}=27$ arrangements which can be clustered into ten different classes [21]. Experimental 


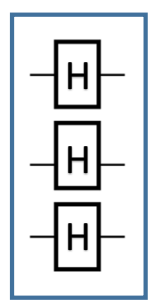

I

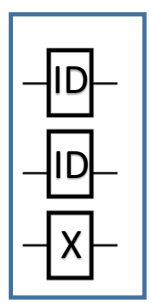

VI

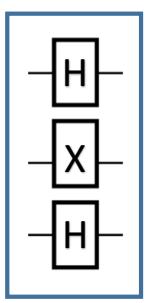

II

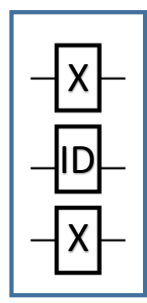

VII

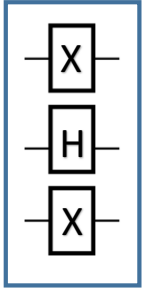

III

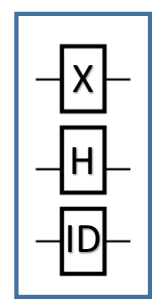

VIII

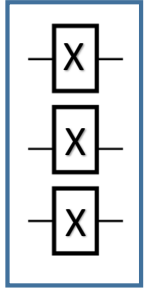

IV

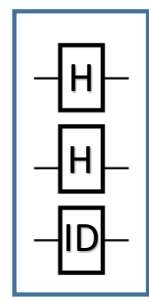

IX

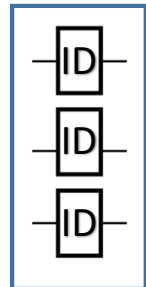

v

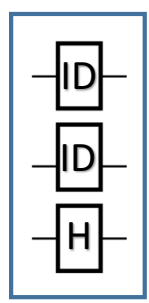

$\mathrm{X}$

Fig. 2 (Color online) Ten classes with different operations used by each player in $S_{1} \otimes S_{2} \otimes S_{3}$. Here, $H$, $X$ and ID are Hadamard, NOT and identity gates, respectively

design for the implementation of all these classes is shown in Fig. 2. Classes I, IV and V have $\left(\begin{array}{l}3 \\ 3\end{array}\right)=1$ configuration, while Classes II, III, VI, VII, IX and X each have $\left(\begin{array}{l}3 \\ 1\end{array}\right)=3$ possible configurations, and there are $3 !=6$ configurations in Class VIII. When each player decides to play a unique tactic, independent of the other players, we obtain class VIII as mixed-strategy Nash Equilibrium. Class VII provides the best response for each player but is not considered as it is biased. The Nash equilibrium is a combination of actions or moves $\left\{\sigma_{A}, \sigma_{B}\right\}$ such that neither party can enhance her payoff $\$_{j}\left(\sigma_{A}, \sigma_{B}\right)$ above the equilibrium value by making a unilateral change of action:

$$
\begin{aligned}
& \$_{A}\left(\sigma_{A}, \sigma_{B}\right) \geq \$\left(\sigma_{A}^{\prime}, \sigma_{B}\right), \\
& \$_{B}\left(\sigma_{A}, \sigma_{B}\right) \geq \$\left(\sigma_{A}, \sigma_{B}^{\prime}\right),
\end{aligned}
$$

where $\sigma_{A}^{\prime}$ is any other strategy of player $A$ than the equilibrium one, and analogously for $\sigma_{B}^{\prime}$.

Suppose the umpire has provided tainted qubits from the black box; i.e., instead of $|000\rangle$, he introduces error (mixedness) of the form $\rho_{0}(x)=(1-x)|000\rangle\langle 000|+$ $x|111\rangle\langle 111|$. The expected payoff would change quite exorbitantly as we increase the amount of preparation noise or corruption (as shown in Fig. 7), where ' $x$ ' is the noise parameter, $x \in[0,1]$.

In IBM implementation [40] of the gate, we performed the entangling gate $J$ using a $R_{x}\left(-\frac{\pi}{2}\right)$ and four CNOT gates as

$$
J=\left(\mathrm{ID}_{0} \otimes C N O T_{1 \rightarrow 2}\right)\left(C N O T_{1 \rightarrow 0} \otimes \mathrm{ID}_{2}\right)\left(\mathrm{ID}_{0} \otimes R_{x}\left(-\frac{\pi}{2}\right)_{1} \otimes \mathrm{ID}_{2}\right)
$$




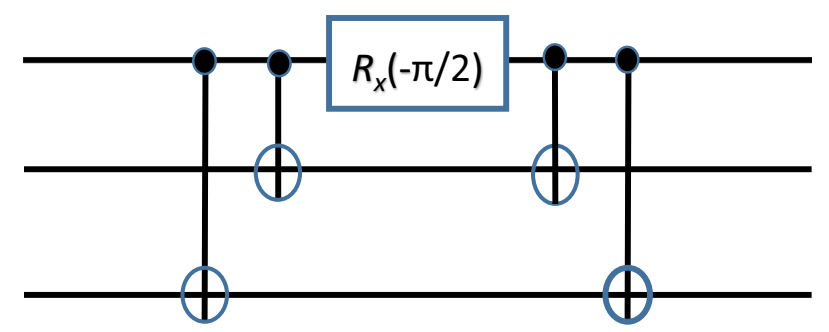

Fig. 3 (Color online) Decomposition of three-qubit operation $J$

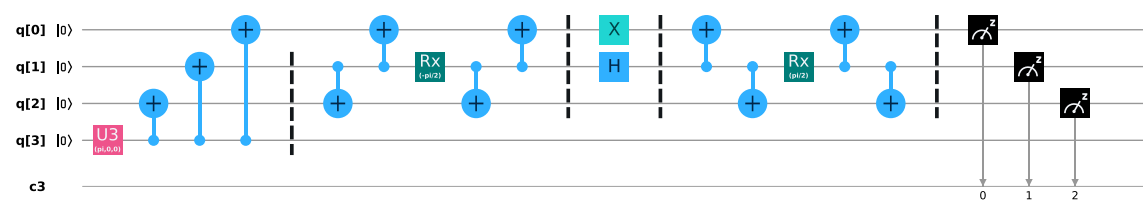

Fig. 4 (Color online) Circuits for the simulation of noisy three-player quantum dilemma game for class VIII as implemented on IBM quantum experience

$$
\left(\mathrm{ID}_{0} \otimes C N O T_{1 \rightarrow 2}\right)\left(C N O T_{1 \rightarrow 0} \otimes \mathrm{ID}_{2}\right)
$$

on qubits 0,1 and 2, respectively (also shown in Fig. 3). The single-qubit operation can be defined as $R_{x}\left(-\frac{\pi}{2}\right)|0\rangle \rightarrow(|0\rangle+i|1\rangle) / \sqrt{2}$. In fact, many simulations of this game have modeled the entangling gate $J$ incorrectly in the past [41] as those matrix decompositions were not the same as $J$. As already discussed, this is followed by the players applying their operations on their respective qubits from the strategy space $S$. Finally, we apply a disentangling gate $J^{\dagger}$ which can be modeled by the same set of gates which was used to construct $J$ since $(A B C D E)^{\dagger}=E^{\dagger} D^{\dagger} C^{\dagger} B^{\dagger} A^{\dagger}$.

To introduce the corruption in the input qubits (shown in Fig. 4), the umpire uses an ancilla in state $|\psi\rangle=U(\theta, \phi, \lambda)|0\rangle=\cos \frac{\theta}{2}|0\rangle+\sin \frac{\theta}{2}|1\rangle$ prepared using singlequbit unitary operation $U(\theta, \phi=0, \lambda=0)$ defined as

$$
U(\theta, \phi, \lambda)=\left(\begin{array}{cc}
\cos \frac{\theta}{2} & -e^{i \lambda} \sin \frac{\theta}{2} \\
e^{i \phi} \sin \frac{\theta}{2} e^{i \lambda+i \phi} \cos \frac{\theta}{2}
\end{array}\right) .
$$

Subsequently, the umpire uses this ancilla as control and applies CNOT gates to the rest of the qubits which he sends to three players. The amount of corruption $x$ is related by $x=\sin ^{2} \frac{\theta}{2}$. Therefore, in what follows, we trace out the fourth qubit to obtain the payoffs of noisy game.

Here, it is imperative that due to corruption $x$ the three-qubit state shared by the players is $\rho_{\text {in }}(x)=J \rho_{0}(x) J^{\dagger}$ instead of $\left|\psi_{\text {in }}\right\rangle=J|000\rangle$ shared among them in the ideal case. In the absence of any knowledge about $x$, all the players perform their strategies assuming they have the latter state. As expected, the performance of the game deteriorates as $x$ causes $\rho_{0}(x)$ to deviate more from $\left|\psi_{\text {in }}\right\rangle$. This deviation can be captured by the fidelity $F=\left\langle\psi_{\text {in }}\left|\rho_{\text {in }}\right| \psi_{\text {in }}\right\rangle=1-x$. The corresponding monotonic reduction in the game's performance is discussed below. 
The corruption that we have considered is the one that is easy to implement in the IBM processor, while at the same time reflective of a general noise process. To compare and contrast it with a better studied noise, which arises during a dissipative interaction of a system with its environment, we further consider the effect of amplitude damping noise on the three-qubit entangled state obtained after application of $J$ operator on tainted qubits provided by the umpire, i.e., $\rho_{\text {in }}$. Here, we restrict ourselves to the effect of amplitude damping noise only on the first qubit, which can be defined in the operator-sum approach by

$$
\rho_{\mathrm{AD}}(x, \lambda)=\sum_{j=0}^{1}\left(K_{j}(\lambda) \otimes \mathrm{ID}^{\otimes 2}\right) \rho_{\mathrm{in}}(x)\left(K_{j}^{\dagger}(\lambda) \otimes \mathrm{ID}^{\otimes 2}\right),
$$

where the Kraus operators are

$$
K_{0}(\lambda)=|0\rangle\langle 0|+\sqrt{1-\lambda}| 1\rangle\langle 1| \text { and } K_{1}(\lambda)=\sqrt{\lambda}|0\rangle\langle 1| \text {. }
$$

All three players apply unitary operations on $\rho_{\mathrm{AD}}$ depending upon their strategy and subsequently perform $J^{\dagger}$ on the combined three-qubit state before measuring it in the $Z$ basis. We can obtain the total payoff of the players as the sum of the probability mass function in the $Z$ basis, multiplied with corresponding values of payoffs in Table 1. The average payoff can be obtained by dividing the total payoff by the number of players. The Nash equilibrium with classical players is $(q, q, q)$ as none of the players can improve his payoff by unilaterally changing his strategy, which is termed as classical Nash equilibrium. In contrast, a quantum player can also apply operation $S_{2}$, not available to a classical user, and this gives Class VIII, which happens to yield the Nash equilibrium in this case, i.e., quantum Nash equilibrium.

The classical and quantum Nash equilibria for the corrupt state preparation and the first qubit undergoing amplitude damping can be obtained as

$$
\begin{aligned}
& \$_{\mathrm{cl}}(x, \lambda)=\frac{1}{12}[2 \lambda p-3 q\{\lambda+2(2 x-1) \sqrt{1-\lambda}-2\}], \\
& \$_{\mathrm{qu}}(x, \lambda)=\frac{1}{24}[3 \lambda q-16 n(2 x-1) \sqrt{1-\lambda}-2(\lambda-4) p] .
\end{aligned}
$$

The variation in the payoff for the Nash equilibrium can be observed to fall (shown in Fig. 5) as $\lambda$ increases from 0 for $x<1 / 2$, while for large values of corruption it can be observed to increase. The case $\lambda=0$ corresponds to the noiseless but corrupted state, and here we observe that the payoff decreases with $x$, which can be explained in terms of gradual fall in the fidelity $F$ of the state $\rho_{0}(x)$.

\section{Results and discussion}

We have performed the experiments for all classes and computed payoff from the measurement outcomes in computational basis. We have also obtained the output density matrices to obtain the fidelity between theoretically desired and experimentally reconstructed states. 


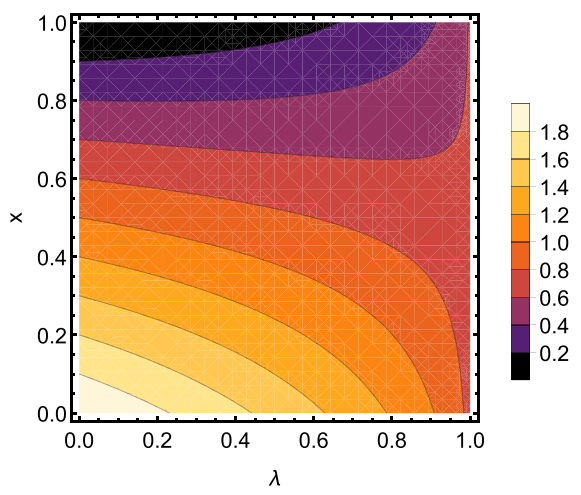

(a)

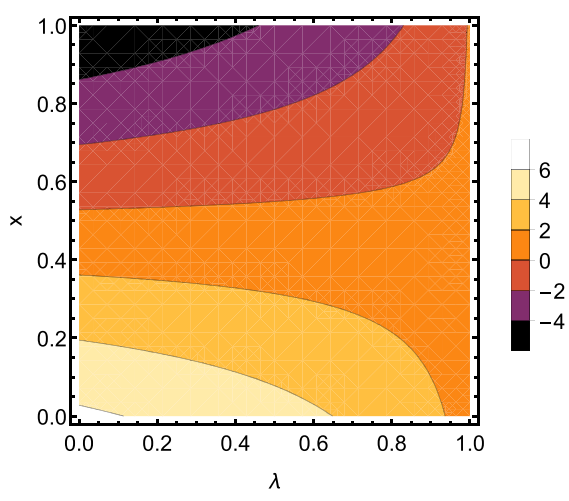

(b)

Fig. 5 (Color online) Variation in average payoff at $\mathbf{a}$ classical and $\mathbf{b}$ quantum Nash equilibria with corruption $x$ and amplitude damping parameter $\lambda$. Here, we have chosen the payoff parameters $n=9, q=2$ and $p=1$

\subsection{Fidelity and quantum state tomography}

In quantum computation, fidelity is used to describe closeness between two states as it is one of the distance-based measures. Ideally, fidelity between the experimental $\left(\rho^{E}\right)$ and theoretical $\left(\rho^{T}\right)$ density matrices, defined as $F\left(\rho^{E}, \rho^{T}\right)=\operatorname{Tr} \sqrt{\left(\sqrt{\rho^{T}} \rho^{E} \sqrt{\left.\rho^{T}\right)}\right.}$ [38], is desired to be 1 , but due to unavoidable errors, it is usually less than unity in most cases.

In our case, we calculate fidelities of all the classes and show them in Table 2. To obtain the experimental density matrices and fidelities, we performed quantum state tomography of the outputs of all the circuits (see $[42,43]$ for detail). The crux of the matter is that we can reconstruct the three-qubit density matrix of the output of the circuit using

$$
\rho^{E}=\frac{1}{2^{3}} \sum_{i_{1}, i_{2}, i_{3}=0}^{3} T_{i_{1} i_{2} i_{3}}\left(\sigma_{i_{1}} \otimes \sigma_{i_{2}} \otimes \sigma_{i_{3}}\right),
$$

where $\sigma_{j}$ are respective Pauli matrices. The values of elements of the $T$ matrix are obtained from the expectation values of these Pauli operators. Specifically, circuit corresponding to each measurement setting is run 8192 times, which is the maximum available number for the device. For instance, in the case of Class VII, $U_{7}=\sigma_{x} \otimes$ ID $\otimes \sigma_{x}$ is the strategy unitary and the experimentally reconstructed density matrix is shown with corresponding theoretical density matrix $\rho_{7}^{T}=|101\rangle\langle 101|$ in Fig. 6. We have also reported experimentally reconstructed and theoretical density matrices for Class VIII with strategy unitary $U_{8}=\sigma_{x} \otimes H \otimes$ ID in Fig. 6. The fidelity of the output density matrices for all the classes is summarized in Table 2. Surprisingly, fidelity and error are not properly anti-correlated; i.e., there are instances where a class of higher fidelity than another still yields a greater error in the payoff. It is worth emphasizing 


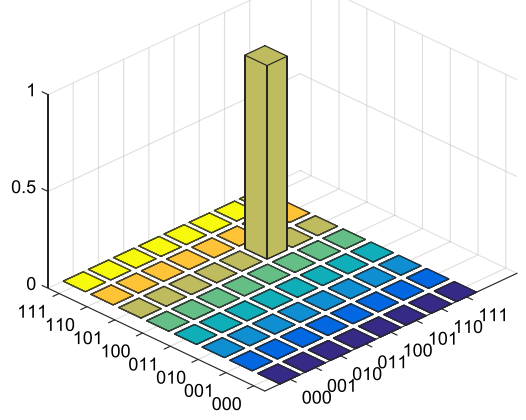

(a)

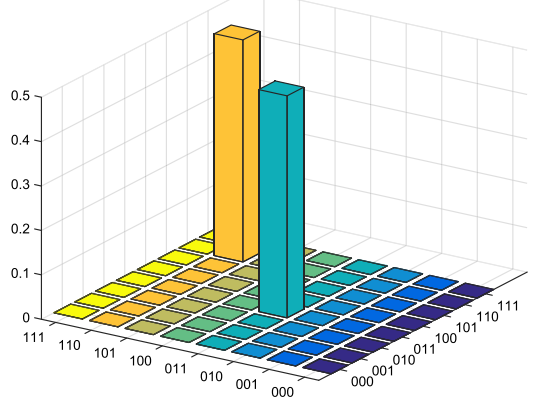

(c)

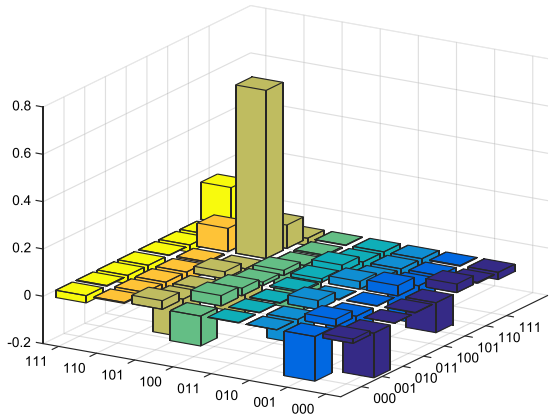

(b)

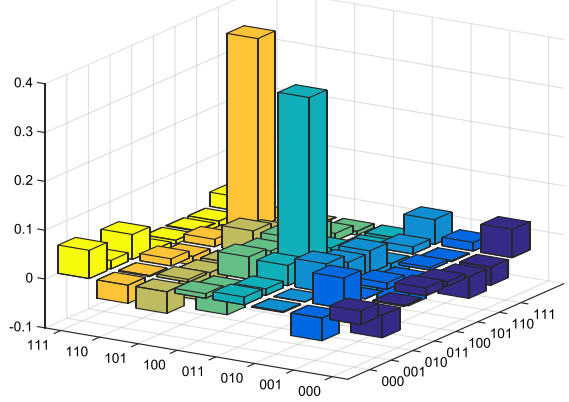

(d)

Fig. 6 (Color online) Graphical representation of $\mathbf{a}$ the theoretical density matrix $\rho_{7}^{T}$ and $\mathbf{b}$ the real part of the experimentally reconstructed density matrix $\operatorname{Re}\left(\rho_{7}^{E}\right)$ for Class VII. Similarly, $\mathbf{c}$ the theoretical matrix $\rho_{8}^{T}$ and $\mathbf{d}$ the real part of the experimentally reconstructed density matrix $\operatorname{Re}\left(\rho_{8}^{E}\right)$ for Class VIII are also shown

here that the payoff readout involves only measurement in the Pauli $\sigma_{z}$ basis, whereas the fidelity is determined via a state tomography, which requires the full suite of Pauli $\sigma_{x}, \sigma_{y}$ and $\sigma_{z}$ measurements. We suspect that the discrepancy between the fidelity and payoff error may be attributed to situation that the gate fidelity of Pauli $\sigma_{z}$ differs from that of Pauli $\sigma_{x}$ or $\sigma_{y}$, on a given processor for certain experimental configurations. Upon inspection, one finds that this discrepancy is not consistently attributable to any one of the processors and may have to do with the behavior of the processor in the context of the full circuit for the experimental configuration. We think this may be an interesting problem for future study in order to compare IBM Q Experience's output with that from other experimental setups. Here, it may be noted that the experiments are performed on different processors provided by IBM depending upon their availability.

\subsection{Payoff table}

Payoff of single player $\$$ is obtained by multiplying their respective payoffs from Table 1 with the probabilities obtained from the output of the experiment (as in [24]). The 


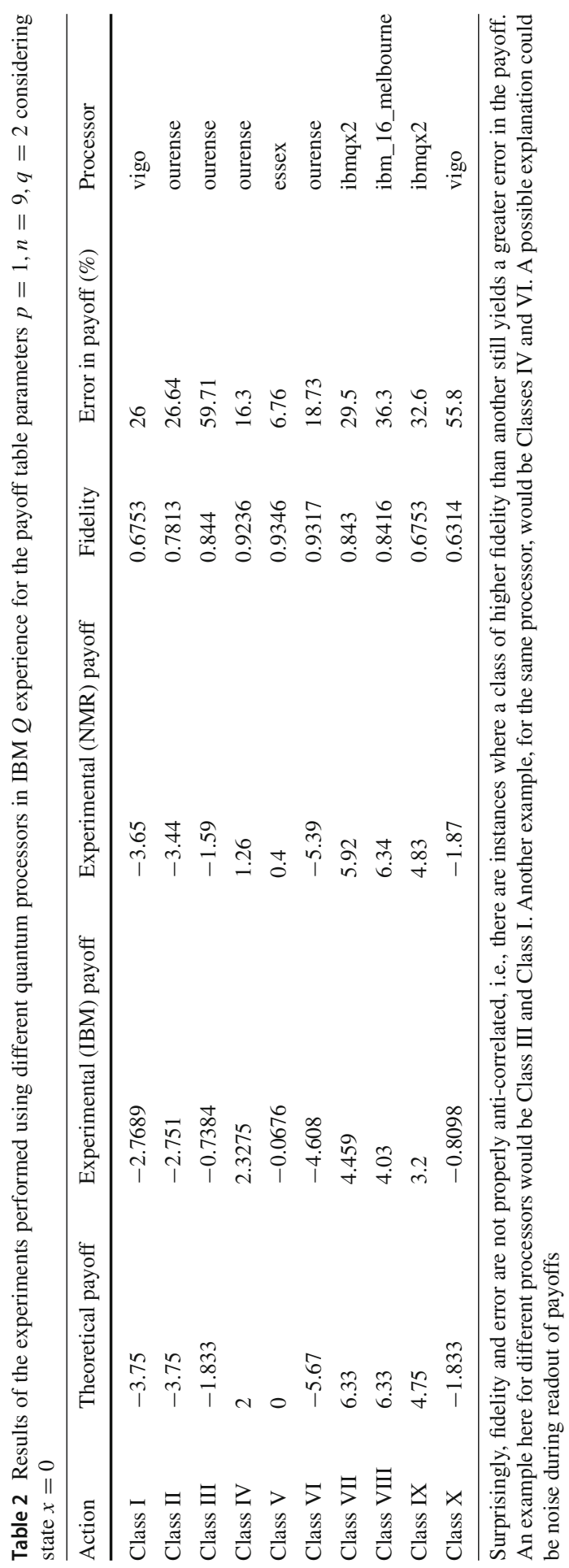


mean payoff per player $\langle \$\rangle$ is defined as the numerical mean of the payoffs of each player $\langle \$\rangle=\frac{\$ 1+\$ 2+\$ 3}{3}$.

It is also shown in the past that the payoffs for quantum Nash equilibrium deteriorate with noise [21]. However, in our case, assuming arbitrary values of parameters, we obtained that quantum Nash equilibrium is $3 \$_{\mathrm{qu}}=-4 n x+2 n+p$, and classical Nash equilibrium for $(q, q, q)$ is $\$_{\mathrm{cl}}=q(1-x)$. As both quantum and classical Nash equilibrium values decrease with corruption level $x$, the quantum advantage disappears after the point of intersection of these two curves. That intersection can be obtained as the critical value of corruption

$$
x_{c}=\frac{2 n+p-3 q}{4 n-3 q} \text {. }
$$

From Fig. 7, we obtain that experimentally $x_{c}=0.363$, whereas the theoretical value is 0.428 [21], giving us an error of $15.18 \%$. Note that the results obtained in [21] neglect decoherence after the initial state is prepared by the demon. However, here on top of that, gate errors in the implementation of the presently available SQUIDbased quantum computing facilities also play an important role in sabotaging the quantum advantage achievable in quantum games. Of course, a reduction in noise with improvement in technology will improve the outcome. Notice that for very high values of corruption, when classical strategy is a preferred choice, the experimental results show higher payoffs than the theoretically expected in quantum Nash equilibrium. This can be understood by interpreting the experimental result as including the effect of decoherence on the ideal quantum state of the processor. This leads in this regime $(x>0.5)$ to a mixture of the theoretical prediction (having a lower value) and the classical prediction (having a higher value). The experimental higher payoff may then be attributed, paradoxically, to the classicalization of the physical state of the quantum processor. This idea is also reflected in our results pertaining to the AD channel.

\subsection{Variation in $p, q, n$}

As we have discussed the general case of the game with arbitrary values of the individual payoff parameters, here we discuss the role of each of these parameters (assuming $0<p<q<n$ ) on $x_{c}$. This would allow us to choose suitable payoff parameters if the noise level $x$ is known, or if they cannot be varied, then to decide whether to employ the quantum or classical game for the problem in a practical situation.

We find that $x_{c}$ increases if $n$ is increased for a constant value of $p$ and $q$. It implies that when the stakes of a game are high (large $n$ ), such that reward for winnings and the amount of losses are very high, the quantum strategies are better in spite of corruption. Further increasing $n$ saturates $x_{c}$ to 0.5 , which signifies that no matter what, if corruption is higher than $50 \%$, classical strategy will always outperform the quantum strategy. The results obtained are shown in Fig. 8a. Here, it is worth noting that whilst it is generally acknowledged that the quantum advantage disappears under sufficiently high noise, a surprise in the present case is that, for the considered scenario, the quantum strategy becomes particularly disadvantageous. This underscores the 


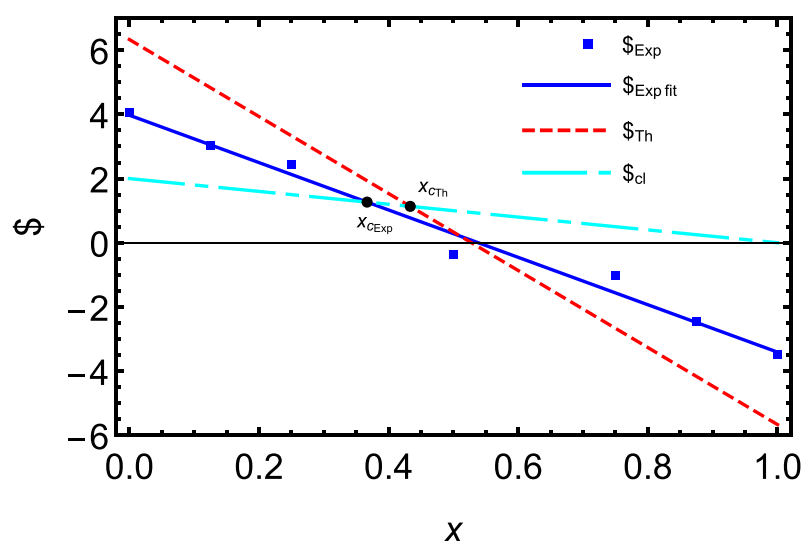

Fig. 7 (Color online) Variation in payoffs in classical and quantum Nash equilibrium with corruption $x$. We have also shown the experimentally obtained payoff values. We assumed $p=1, n=9$ and $q=2$. Here, $x_{C_{\mathrm{Th}}}$ and $x_{c_{\mathrm{Exp}}}$ represent critical values of corruption from theoretical and experimental results. Noise degrades quantum information, and thus not surprisingly, $x_{c_{\mathrm{Th}}}>x_{c_{\mathrm{Exp}}}$

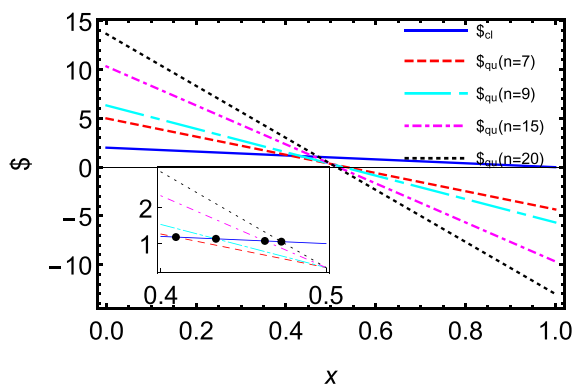

(a)

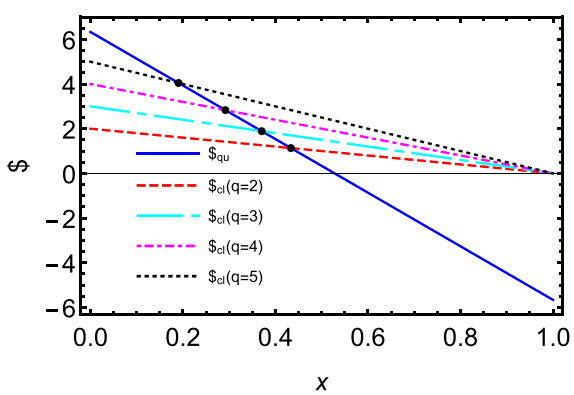

(b)

Fig. 8 (Color online) Variation in payoffs in classical and quantum strategies with corruption $x$ and payoff parameters a $n(q=2)$ and $\mathbf{b} q(n=9)$ with $p=1$ for Class VIII considering rest of the parameters in $\{p, n, q\}$ as constant. The critical value of corruption $x_{c}$ is marked by black dots in all cases

importance of characterizing the noisy channel that determines the level of preparation corruption $x$. A full quantum process tomography of the channel is not necessary, and even a partial one (e.g., Ref. [44]) that allows us to estimate the degree of corruption will suffice.

Note that the only quantum Nash equilibrium depends upon $n$, while classical Nash equilibrium is a function of $q$. Thus, an increase in $q$ essentially leads to classical dominant strategy; i.e., classical strategy tends to be as efficient as the quantum strategy [cf. in Fig. 8b]. However, for large values of corruption, there is no evident advantage as for maximum corruption classical Nash equilibrium is always zero.

These observations lead to conclusion that quantum systems are more prone to errors and deteriorate rapidly with an increase in the amount of corruption. Hence, errors in system may lead to loss of quantum advantage originally present as observed 
from the experimental value in Fig. 7 as well. Thus, in the case of high errors, it is always better to stick to classical strategies from an outsider's perspective.

\section{Conclusion}

We have discussed a multiparty quantum game by generalizing the payoff table. Our result may find interesting applications in diverse fields, such as finance, social networks. Suppose a group of companies wants to invest in a particular stock and has limited knowledge of the market statistics, then the financial situation of the stock simulates a general case of three-person dilemma with arbitrary payoff parameters. In this case, if the stakes of the investments are high such that returns are great, but so are the losses, companies perform better if they use quantum strategies, provided the amount of preparation noise is less than $50 \%$. Otherwise, the classical strategy should be preferred. Similarly, in the situation that the classical dominant strategy equilibrium $(q, q, q)$ has relatively higher payoff than all other cases, the present results may persuade companies to opt for classical strategies even for a small amount of source error. Also note that the mixed strategy, i.e., 50\% chance of investing in the present case, would only have advantage with quantum strategies, i.e., in a low-corruption situation.

We have performed an experiment for the noisy three-player quantum dilemma game and observed that the obtained results were less robust against noise than the corresponding results from NMR experiments. Further, it can be observed that due to additional errors (other than source error introduced in the noisy counterpart of the game), the advantage of quantum game over corresponding classical game disappears quickly. Similar studies for the generalizations of other games where quantum players perform better or the games where classical strategies are always preferable can be performed to study the role of various payoff parameters in those cases. The present experimental implementation of the noisy quantum game on a small noisy quantum computer establishes a practical quantum advantage in game theory. However, in view of noisy intermediate-scale quantum (NISQ) technology [45] around the corner, i.e., quantum computing infrastructure with 50-100 qubits, this advantage can be exploited for several applications, such as in quantum machine learning [46]. This can be further extended to the iterated version of the game where it is performed more than once, and rational players decide their strategies depending upon their opponents' previous decision. The results from the experiment performed on NMR were more accurate, showing that the NMR-based quantum computer is less noisy. To obtain a quantitative perception of that, we performed quantum state tomography here, which shows that higher fidelity of experimentally generated state does not necessarily mean smaller errors; i.e., fidelity and errors are not properly anti-correlated.

In the end, we would like to stress on the recent studies connecting Bell nonlocality [47,48], a quantum secure direct communication scheme [49] and security of quantum key distribution schemes [50] with game theory. In view of these works, in principle, all quantum cryptographic schemes (see [51,52] for a review) can be viewed from the perspective of game theory, as a game to perform cryptanalysis and obtain security proofs. For example, measurement-device-independent, device-independent 
and entangled state-based quantum key distribution schemes, such as Ekert's scheme [53], can be viewed as a three-party game involving Alice, Bob and Eve. Future work is planned to rigorously analyze the best strategy of Alice and Bob and that of Eve using a game theoretic approach. We hope the present results will be helpful in the application of quantum strategies in game theory, and in turn in their applications in quantum technologies in general, and quantum cryptography in particular.

Acknowledgements AP and RS acknowledge the support from the QUEST scheme of Interdisciplinary Cyber Physical Systems (ICPS) program of the Department of Science and Technology (DST), India (Grant No.: DST/ICPS/QuST/Theme-1/2019/14). KT acknowledges the financial support from the Operational Programme Research, Development and Education, European Regional Development Fund (Project No. CZ.02.1.01/0.0/0.0/16_019/0000754) of the Ministry of Education, Youth and Sports of the Czech Republic. Authors also thank Prof. Anil Kumar and Dr. Abhishek Shukla for their interest and technical comments on this work.

\section{Appendix: Reconstructed density matrix}

The real and imaginary parts of the experimentally obtained density matrix for Class VII by performing quantum state tomography are

$$
\operatorname{Re}\left(\rho_{7}^{E}\right)=\left[\begin{array}{cccccccc}
0.018 & -0.188 & -0.001 & 0.002 & -0.126 & 0.036 & -0.004 & -0.031 \\
-0.188 & 0.030 & -0.100 & 0.002 & 0.044 & -0.160 & -0.019 & -0.003 \\
-0.001 & -0.100 & 0.037 & -0.003 & 0.033 & -0.056 & -0.049 & -0.035 \\
0.002 & 0.002 & -0.003 & 0.007 & 0.015 & 0.030 & -0.042 & -0.039 \\
-0.126 & 0.044 & 0.033 & 0.015 & 0.022 & -0.189 & -0.006 & -0.002 \\
0.036 & -0.160 & -0.056 & 0.030 & -0.189 & 0.711 & 0.099 & -0.030 \\
-0.004 & -0.019 & -0.049 & -0.042 & -0.006 & 0.099 & 0.018 & -0.022 \\
-0.031 & -0.003 & -0.035 & -0.039 & -0.002 & -0.030 & -0.022 & 0.157
\end{array}\right]
$$

and

$$
\operatorname{Im}\left(\rho_{7}^{E}\right)=\left[\begin{array}{cccccccc}
0 & 0.229 & 0.002 & 0.009 & 0.142 & -0.042 & -0.028 & -0.009 \\
-0.229 & 0 & 0 & -0.001 & -0.001 & 0.148 & -0.010 & -0.034 \\
-0.002 & 0 & 0 & 0.002 & -0.011 & 0.014 & 0.053 & 0.010 \\
-0.009 & 0.001 & -0.002 & 0 & 0.014 & 0.017 & 0.004 & 0.030 \\
-0.142 & 0.001 & 0.011 & -0.014 & 0 & 0.158 & 0.004 & -1.108 \\
0.042 & -0.148 & -0.014 & -0.017 & -0.158 & 0 & -1.103 & 0.058 \\
0.028 & 0.010 & -0.053 & -0.004 & -0.004 & 1.103 & 0 & 0.024 \\
0.009 & 0.034 & -0.010 & -0.030 & 1.108 & -0.058 & -0.024 & 0
\end{array}\right]
$$

respectively, while the theoretical density matrix in the corresponding case is given by $\rho_{7}^{T}=\left|\psi_{7}\right\rangle\left\langle\psi_{7}|=| 101\right\rangle\langle 101|$, where $\left|\psi_{7}\right\rangle=J^{\dagger} \cdot U_{7} \cdot J|000\rangle$. Similarly, the real and imaginary parts of the experimentally obtained density matrix for Class VIII by performing quantum state tomography are 


$$
\operatorname{Re}\left(\rho_{8}^{E}\right)=\left[\begin{array}{ccccccccc}
0.028 & -0.046 & 0.002 & 0.016 & 0.01 & -0.044 & -0.037 & 0.058 \\
-0.046 & 0.062 & 0.000 & 0.013 & -0.053 & 0.006 & -0.002 & 0.018 \\
0.002 & 0.000 & 0.059 & 0.042 & 0.046 & -0.06 & 0.015 & 0.051 \\
0.016 & 0.013 & 0.042 & 0.366 & 0.038 & -0.025 & -0.017 & -0.011 \\
0.01 & -0.053 & 0.046 & 0.038 & 0.046 & 0.046 & 0.015 & -0.008 \\
-0.044 & 0.006 & -0.06 & -0.025 & 0.046 & 0.023 & -0.016 & -0.002 \\
-0.037 & -0.002 & 0.015 & -0.017 & 0.015 & -0.016 & 0.387 & -0.022 \\
0.058 & 0.018 & 0.051 & -0.011 & -0.008 & -0.002 & -0.022 & 0.029
\end{array}\right]
$$

and

$$
\operatorname{Im}\left(\rho_{8}^{E}\right)=\left[\begin{array}{cccccccc}
0 & -0.001 & -0.005 & 0 & -0.014 & 0 & -0.022 & -0.077 \\
0.001 & 0 & 0.005 & -0.107 & -0.028 & 0.002 & -0.083 & 0.003 \\
0.005 & -0.005 & 0 & 0.004 & 0.003 & 0.087 & 0.009 & -0.003 \\
0 & 0.107 & -0.004 & 0 & 0.052 & 0.015 & -0.331 & -0.016 \\
0.014 & 0.028 & -0.003 & -0.052 & 0 & 0 & -0.068 & 0.011 \\
0 & -0.002 & -0.087 & -0.015 & 0 & 0 & 0.011 & 0 \\
0.022 & 0.083 & -0.009 & 0.331 & 0.068 & -0.016 & 0 & -0.026 \\
0.077 & -0.003 & 0.003 & 0.016 & -0.011 & 0 & 0.026 & 0
\end{array}\right],
$$

respectively, while the theoretical density matrix in the corresponding case is given by $\rho_{8}^{T}=\left|\psi_{8}\right\rangle\left\langle\psi_{8}\right|$, where $\left|\psi_{8}\right\rangle=J^{\dagger} \cdot U_{8} \cdot J|000\rangle$.

\section{References}

1. Flitney, A.P., Abbott, D.: An introduction to quantum game theory. Fluct. Noise Lett. 2, R175-R187 (2002)

2. Piotrowski, E.W., Sładkowski, J.: An invitation to quantum game theory. Int. J. Theor. Phys. 42, 10891099 (2003)

3. Li, X., Gao, L., Li, W.: Application of game theory based hybrid algorithm for multi-objective integrated process planning and scheduling. Exp. Syst. Appl. 39, 288-297 (2012)

4. Elhenawy, M., Elbery, A.A., Hassan, A.A., Rakha, H.A.: An intersection game-theory-based traffic control algorithm in a connected vehicle environment. In: 2015 IEEE 18th international conference on intelligent transportation systems, pp. 343-347. IEEE (2015)

5. Laszka, A., Szeszlér, D., Buttyán, L.: Game-theoretic robustness of many-to-one networks. In: International Conference on Game Theory for Networks, pp. 88-98. Springer (2012)

6. Dodis, Y., Rabin, T., et al.: Cryptography and game theory. Algorithmic game theory, pp. 181-207. (2007)

7. Li, A., Yong, X.: Entanglement guarantees emergence of cooperation in quantum prisoner's dilemma games on networks. Sci. Rep. 4, 1-7 (2014)

8. Nawaz, A., Toor, A.H.: Dilemma and quantum battle of sexes. J. Phys. A Math. Gen. 37, 4437 (2004)

9. Meyer, D.A.: Quantum strategies. Phys. Rev. Lett. 82, 1052 (1999)

10. Eisert, J., Wilkens, M., Lewenstein, M.: Quantum games and quantum strategies. Phys. Rev. Lett. 83, 3077 (1999)

11. Du, J., Xu, X., Li, H., Zhou, X., Han, R.: Playing prisoner's dilemma with quantum rules. Fluct. Noise Lett. 2, R189-R203 (2002)

12. Werner, R.F.: Optimal cloning of pure states. Phys. Rev. A 58, 1827 (1998)

13. Tomamichel, M., Fehr, S., Kaniewski, J., Wehner, S.: A monogamy-of-entanglement game with applications to device-independent quantum cryptography. New J. Phys. 15, 103002 (2013)

14. Fritz, T., Sainz, A.B., Augusiak, R., et al.: Local orthogonality as a multipartite principle for quantum correlations. Nat. Commun. 4, 1 (2013) 
15. Oppenheim, J., Wehner, S.: The uncertainty principle determines the nonlocality of quantum mechanics. Science 330, 1072 (2010)

16. Anshu, A., Høyer, P., Mhalla, M., Perdrix, S.: Contextuality in multipartite pseudo-telepathy graph games. J. Comput. Syst. Sci. 107, 156-165 (2020)

17. Popescu, S.: Nonlocality beyond quantum mechanics. Nat. Phys. 10, 264-270 (2014)

18. Chen, C., Dong, D., Dong, Y., Shi, Q.: A quantum reinforcement learning method for repeated game theory. In: 2006 International Conference on Computational Intelligence and Security volume 1, pp. 68-72. IEEE (2006)

19. Clausen, J., Briegel, H.J.: Quantum machine learning with glow for episodic tasks and decision games. Phys. Rev. A 97, 022303 (2018)

20. Benjamin, S.C., Hayden, P.M.: Multiplayer quantum games. Phys. Rev. A 64, 030301 (2001)

21. Johnson, N.F.: Playing a quantum game with a corrupted source. Phys. Rev. A 63, 020302 (2001)

22. Daskalakis, C., Papadimitriou, C.H.: Three-player games are hard. Electr. Colloq. Comput. Complex. 139, 81-87 (2005)

23. Daskalakis, C., Goldberg, P.W., Papadimitriou, C.H.: The complexity of computing a Nash equilibrium. SIAM J. Comput. 39, 195-259 (2009)

24. Mitra, A., Sivapriya, K., Kumar, A.: Experimental implementation of a three qubit quantum game with corrupt source using nuclear magnetic resonance quantum information processor. J. Magn. Reson. 187, 306-313 (2007)

25. Kolenderski, P., Sinha, U., Youning, L., et al.: Aharon-Vaidman quantum game with a Young-type photonic qutrit. Phys. Rev. A 86, 012321 (2012)

26. Pinheiro, A., Souza, C., Caetano, D., et al.: Vector vortex implementation of a quantum game. JOSA B 30, 3210-3214 (2013)

27. Schmid, C., Flitney, A.P., Wieczorek, W., et al.: Experimental implementation of a four-player quantum game. New J. Phys. 12, 063031 (2010)

28. Zhou, L., Kuang, L.-M.: Proposal for optically realizing a quantum game. Phys. Lett. A 315, 426-430 (2003)

29. Solmeyer, N., Linke, N.M., Figgatt, C., et al.: Demonstration of a Bayesian quantum game on an ion-trap quantum computer. Quantum Sci. Technol. 3, 045002 (2018)

30. Glazer, J., Ma, C.-T.A.: Efficient allocation of a "prize”-King Solomon's dilemma. Games Econ. Behav. 1, 222-233 (1989)

31. Teng, Y., Jones, R., Marusich, L., et al.: Trust and situation awareness in a 3-player diner's dilemma game. In: 2013 IEEE International Multi-Disciplinary Conference on Cognitive Methods in Situation Awareness and Decision Support (CogSIMA), pp. 9-15. IEEE (2013)

32. Bankes, S.: Exploring the foundations of artificial societies: Experiments in evolving solutions to iterated N-player prisoner's dilemma. In: Artificial Life IV, pp. 337-342. MIT Press Cambridge, MA (1994)

33. Morgan, J.P., Chaganty, N.R., Dahiya, R.C., Doviak, M.J.: Let's make a deal: the player's dilemma. Am. Stat. 45, 284-287 (1991)

34. Özdemir, Ş.K., Shimamura, J., Imoto, N.: Quantum advantage does not survive in the presence of a corrupt source: optimal strategies in simultaneous move games. Phys. Lett. A 325, 104-111 (2004)

35. Chen, J.-L., Kwek, L.C., Oh, C.H.: Noisy quantum game. Phys. Rev. A 65, 052320 (2002)

36. Chen, L., Ang, H., Kiang, D., Kwek, L., Lo, C.: Quantum prisoner dilemma under decoherence. Phys. Lett. A 316, 317-323 (2003)

37. Sisodia, M., Shukla, A., Thapliyal, K., Pathak, A.: Design and experimental realization of an optimal scheme for teleportation of an n-qubit quantum state. Quantum Inform. Process. 16, 292 (2017)

38. Sisodia, M., Shukla, A., de Almeida, A.A., Dueck, G.W., Pathak, A.: Circuit optimization for IBM processors: A way to get higher fidelity and higher values of nonclassicality witnesses. arXiv preprint arXiv:1812.11602 (2018)

39. Tian, J., Uchida, N.: Monkeys in a prisoner's dilemma. Cell 160, 1046-1048 (2015)

40. Shukla, A., Sisodia, M., Pathak, A.: Complete characterization of the directly implementable quantum gates used in the IBM quantum processors. arXiv preprint arXiv:1805.07185 (2018)

41. Sousa, P.B., Ramos, R.V.: Universal quantum circuit for n-qubit quantum gate: A programmable quantum gate. arXiv preprint arXiv:quant-ph/0602174 (2006)

42. Sisodia, M., Shukla, A., Pathak, A.: Experimental realization of nondestructive discrimination of Bell states using a five-qubit quantum computer. Phys. Lett. A 381, 3860-3874 (2017) 
43. Vishnu, P., Joy, D., Behera, B.K., Panigrahi, P.K.: Experimental demonstration of non-local controlledunitary quantum gates using a five-qubit quantum computer. Quantum Inform. Process. 17, 274 (2018)

44. Mohseni, M., Lidar, D.A.: Direct characterization of quantum dynamics. Phys. Rev. Lett. 97, 170501 (2006)

45. Preskill, J.: Quantum computing in the NISQ era and beyond. Quantum 2, 79 (2018)

46. Torlai, G., Melko, R.: Machine-learning quantum states in the NISQ era. Ann. Rev. Condens. Matter Phys. 11, 325 (2019)

47. Brunner, N., Linden, N.: Connection between Bell nonlocality and Bayesian game theory. Nat. Commun. 4, 2057 (2013)

48. Iqbal, A., Chappell, J.M., Abbott, D.: The equivalence of Bell's inequality and the Nash inequality in a quantum game-theoretic setting. Phys. Lett. A 382, 2908-2913 (2018)

49. Kaur, H., Kumar, A.: Game-theoretic perspective of ping-pong protocol. Phys. A Stat. Mech Its Appl. 490, 1415-1422 (2018)

50. Krawec, W. O., Miao, F.: Game theoretic security framework for quantum key distribution. In: International Conference on Decision and Game Theory for Security, pp. 38-58. Springer (2018)

51. Pathak, A.: Elements of Quantum Computation and Quantum Communication. Taylor \& Francis, New York (2013)

52. Thapliyal, K., Pathak, A., Banerjee, S.: Quantum cryptography over non-Markovian channels. Quantum Inform. Process. 16, 115 (2017)

53. Ekert, A.K.: Quantum cryptography based on Bell's theorem. Phys. Rev. Lett. 67, 661 (1991)

Publisher's Note Springer Nature remains neutral with regard to jurisdictional claims in published maps and institutional affiliations. 